\title{
Superconducting and charge-density wave instabilities in ultrasmall-radius carbon nanotubes
}

\section{Citation}

Barnett, Ryan, Eugene Demler, and Efthimios Kaxiras. 2005. "Superconducting and Chargedensity Wave Instabilities in Ultrasmall-Radius Carbon Nanotubes." Solid State Communications 135 (5): 335-39. https://doi.org/10.1016/j.ssc.2005.04.049.

\section{Permanent link}

http://nrs.harvard.edu/urn-3:HUL.InstRepos:41384127

\section{Terms of Use}

This article was downloaded from Harvard University's DASH repository, and is made available under the terms and conditions applicable to Other Posted Material, as set forth at http:// nrs.harvard.edu/urn-3:HUL.InstRepos:dash.current.terms-of-use\#LAA

\section{Share Your Story}

The Harvard community has made this article openly available.

Please share how this access benefits you. Submit a story.

Accessibility 


\title{
Superconducting and charge-density wave instabilities in ultrasmall-radius carbon nanotubes
}

\author{
Ryan Barnett, Eugene Demler, and Efthimios Kaxiras \\ Department of Physics, Harvard University, Cambridge MA 02138
}

(Dated: November 16, 2018)

\begin{abstract}
We perform a detailed analysis of the band structure, phonon dispersion, and electron-phonon coupling of three types of small-radius carbon nanotubes (CNTs): $(5,0),(6,0)$, and $(5,5)$ with diameters $3.9,4.7$, and $6.8 \AA$ respectively. The large curvature of the $(5,0)$ CNTs makes them metallic with a large density of states at the Fermi energy. The density of states is also strongly enhanced for the $(6,0)$ CNTs compared to the results obtained from the zone-folding method. For the $(5,5)$ CNTs the electron-phonon interaction is dominated by the in-plane optical phonons, while for the ultrasmall $(5,0)$ and $(6,0)$ CNTs the main coupling is to the out-of-plane optical phonon modes. We calculate electron-phonon interaction strengths for all three types of CNTs and analyze possible instabilities toward superconducting and charge-density wave phases. For the smallest $(5,0)$ nanotube, in the mean-field approximation and neglecting Coulomb interactions, we find that the charge-density wave transition temperature greatly exceeds the superconducting one. When we include a realistic model of the Coulomb interaction we find that the charge-density wave is suppressed to very low temperatures, making superconductivity dominant with the mean-field transition temperature around one K. For the $(6,0)$ nanotube the charge-density wave dominates even with the inclusion of Coulomb interactions and we find the mean-field transition temperature to be around five Kelvin. We find that the larger radius $(5,5)$ nanotube is stable against superconducting and charge-density wave orders at all realistic temperatures.
\end{abstract}

The discovery of carbon nanotubes [1] has lead to a renewed interest in the study of $1 \mathrm{~d}$ electron systems. The difference between semiconducting and metallic largeradius nanotubes may be typically understood by quantizing the circumferential momentum of the electronic states in a single graphene sheet (see, for instance, 2]). Less conventional properties of nanotubes include Luttinger liquid behavior of metallic nanotubes found in tunneling experiments (see [3] and references therein), Coulomb effects [4], Kondo physics 5], and intrinsic superconductivity observed in ropes [6] and small-radius nanotubes in a zeolite matrix 7]. The main focus has traditionally been on the effects of the Coulomb interaction between electrons. However, the electron-phonon interaction has also received considerable attention both experimentally [8, [9] and theoretically [10, 11, 12]. Most theoretical analyses of electron-phonon interactions in nanotubes assume the phonon frequencies to be the same as in a graphene sheet and calculate the electron-phonon coupling strength from a simplified tight-binding model for the $\pi$ orbitals of the $\mathrm{C}$ atoms. Such an approach, however, may not be suited for ultrasmall nanotubes (such as the ones in [7]), for which the curvature of the nanotube leads to strong hybridization of the $\sigma$ and $\pi$ orbitals, which results in a qualitatively different band structure [13], phonon spectrum, and electron-phonon interactions.

In this Letter we present detailed analysis of three representative small-radius nanotubes, the $(5,0),(6,0)$, and $(5,5)$, and discuss possible CDW and superconducting instabilities of these systems. The $(5,0)$ nanotube is the likely candidate structure for the superconducting behavior seen in [7] with transition temperature measured around $15 \mathrm{~K}$. The radii of the CNTs in this exper- iment has been determined to within $0.2 \AA$ by Raman spectroscopy [14. We demonstrate that even though standard electronic structure approaches for calculating phonon frequencies, such as the frozen-phonon approximation (FPA), run into divergences intrinsic to meanfield calculations in $1 \mathrm{~d}$, they can be analyzed from the point of view of the random-phase approximation (RPA) for the electron-phonon system and parameters of the effective Frölich Hamiltonian can be extracted. The main results that we obtain are: (i) The strongest electronphonon coupling for the $(5,0)$ and $(6,0)$ CNTs is to the out-of-plane phonon modes. This is in contrast to the larger radius CNTs which have strongest coupling to the in-plane phonon modes as predicted by the nearestneighbor tight-binding model 2]. (ii) Even when the residual Coulomb interaction between electrons is neglected, the larger radius $(5,5)$ CNT remains stable down to extremely low temperatures. For the smaller radii $(5,0)$ and $(6,0)$ CNTs, when the residual Coulomb interaction is neglected, the CDW instability was found to be dominant over superconductivity for both types of nanotubes. (iii) We include the residual Coulomb interaction between electrons following a model developed in Ref. 15]. For the $(6,0) \mathrm{CNT}$ we find that the CDW transition is essentially unaffected by including the Coulomb interaction and we obtain $T_{\mathrm{CDW}}=5 \mathrm{~K}$. By contrast, for the $(5,0)$ CNT both CDW and SC are suppressed but now superconductivity becomes dominant with $T_{\mathrm{SC}}$ around 1 Kelvin.

The interaction between conduction electrons and vibrations of a crystal lattice is commonly described by 
using the Frölich Hamiltonian

$$
\begin{aligned}
\mathcal{H}_{\mathrm{e}-\mathrm{ph}} & =\sum_{k \tau \sigma} \varepsilon_{k \tau} c_{k \tau \sigma}^{\dagger} c_{k \tau \sigma}+\sum_{q \mu} \Omega_{q \mu}^{0}\left(a_{q \mu}^{\dagger} a_{q \mu}+\frac{1}{2}\right) \\
& +\sum_{k \tau k^{\prime} \tau^{\prime} \sigma \mu} g_{k \tau k^{\prime} \tau^{\prime} \mu} c_{k \tau \sigma}^{\dagger} c_{k^{\prime} \tau^{\prime} \sigma}\left(a_{q \mu}+a_{-q \mu}^{\dagger}\right) .
\end{aligned}
$$

Here $c_{k \tau \sigma}^{\dagger}$ creates an electron with quasimomentum $k$ in band $\tau$ with spin $\sigma, a_{q \mu}^{\dagger}$ creates a phonon with lattice momentum $q$ and polarization $\mu$, and $q=k-k^{\prime}$ modulo a reciprocal lattice vector. The energies of electron quasiparticles and phonons in the absence of electron-phonon coupling are given by $\varepsilon_{k \tau}$ and $\Omega_{q \mu}^{0}$ respectively and the electron-phonon vertex is given by $g_{k \tau k^{\prime} \tau^{\prime} \sigma \mu}$.

To compute the quasiparticle energies $\varepsilon_{k \tau}$ of the representative CNTs, we use the NRL tight-binding method [16] which has been tested extensively and provides accurate results on a variety of materials. After fully relaxing the structures with respect to the atomic coordinates, the band structure is calculated. We find the band structure predicted by zone-folding to agree very well with the calculated band structure of the larger radius $(5,5) \mathrm{CNT}$. However, for the smaller radius $(5,0)$ and $(6,0)$ CNTs there was found to be qualitative differences as shown in Fig. 11 While zone-folding arguments predict the $(5,0)$ nanotube to be insulating, the band structure clearly exhibits metallic behavior. The inner band (with the smaller $k_{F}^{A}$ ) is doubly degenerate while the outer band (with the larger $k_{F}^{B}$ ) is nondegenerate where we have the exact relation $2 k_{F}^{A}=k_{F}^{B}$. The failure of the zone-folding procedure is due to the strong curvature effects, which lead to considerable band shifts in small-radius nanotubes, as discussed originally in 13. for $(6,0)$ nanotubes based on density-functional theory calculations. As a result of these band shifts, for the $(5,0)$ nanotubes we have a system close to a Van Hove singularity which has a density of states of 0.16 states / $\mathrm{eV} /$ carbon atom. For comparison, the density of states for the $(5,5)$ nanotube is only 0.028 states / eV / carbon atom, so we expect that instabilities of the electronphonon systems for the ultrasmall nanotubes are strongly enhanced compared to larger radius nanotubes.

The electron-phonon vertex given in Eq. (11) can be expressed as

$$
g_{k \tau k^{\prime} \tau^{\prime} \mu}=\sqrt{\frac{1}{2 \Omega_{q \mu}^{0} M N N_{c}}} M_{k \tau k^{\prime} \tau^{\prime} \mu}
$$

where $M_{k \tau k^{\prime} \tau^{\prime} \mu}=\frac{1}{u}\left\langle\psi_{k \tau}\left|\left(V^{q \mu}-V_{0}\right)\right| \psi_{k^{\prime} \tau^{\prime}}\right\rangle$. Here, $V^{q \mu}$ is the crystal potential under the presence of a phonon specified by the ionic displacements $\delta \mathbf{R}_{n i}=u e^{i q R_{n}} \hat{\epsilon}_{q \mu}(i)$ and $V_{0}$ is the crystal potential at equilibrium. We calculate the magnitude of these matrix elements for the coupling between electrons on the Fermi surface to all phonon modes. For the $(5,0)$ and $(6,0)$ CNTs we find that the strongest coupling are to out-of-plane modes. More

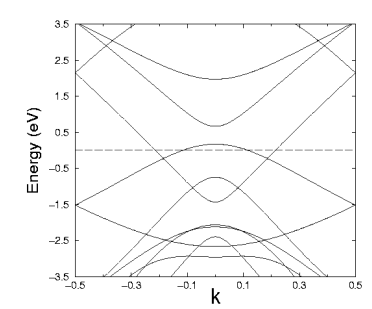

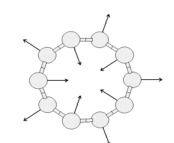

(a)

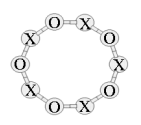

(c)

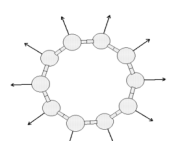

(b)

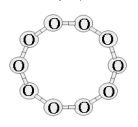

(d)
FIG. 1: Left: The band structure of a $(5,0)$ nanotube where we set $E_{F}=0$. Right: The phonon modes that have the strongest electron-phonon coupling. Shown is a crosssectional slice of the nanotube containing 10 atoms. Modes (a), (b), (c), and (d) are out-of-plane optical, out-of-plane breathing, in-plane optical, and in-plane acoustic modes respectively. The X's and O's denote vectors in and out of the page.

specifically, the strongest overall coupling was found to be to the out-of-plane optical mode followed by the breathing mode which are shown in Fig. 1 This is in contrast to the larger radius $(5,5)$ CNT which has dominant coupling coming from an in-plane optical mode. We point out that in general, the phonon modes of CNTs cannot be classified as in-plane or out-of-plane [2]. However, the modes that have the strongest electron-phonon interactions for the CNTs we study still allow such characterization (see Fig. 1). Moreover, CNT dynamical matrix calculations 17] show that the eigenvectors of these modes are essentially the same as in the graphene sheet.

We point out that, in general, the phonon mode eigenvectors will be influenced by curvature effects, and will differ from the graphene results. We checked the relevant modes by using the CNT dynamical matrix, and found that they agree well with the zone-folding results 17 .

Now we move on to the issue of how to calculate the bare phonon frequencies $\Omega_{q \mu}^{0}$ in the Frölich Hamiltonian. In the standard FPA [18], the frequencies are given by

$$
\Omega_{q \mu}=\frac{1}{u \sqrt{M N_{c}}} \sqrt{\left.\Delta E_{\mathrm{cos}}+\Delta E_{\sin }\right)}
$$

where $u$ is the amplitude of the displacement, and $\Delta E_{\cos }$ and $\Delta E_{\text {sin }}$ are the energy differences per unit cell between the distorted and equilibrium lattice structures where the distortion corresponds to the real and imaginary parts of $\delta \mathbf{R}_{n i}=u e^{i q R_{n}} \hat{\varepsilon}_{q \mu}(i)$ respectively. The phonon dispersion curve for the out-of-plane optical mode obtained from the FPA for the $(5,0)$ CNT is shown in Fig. 2] Unit cell sizes of up to 400 atoms in increments of 20 (which is the number of atoms in the smallest possible unit cell) were used in the FPA, requiring the phonon wave vectors to be commensurate with the chosen supercell. This mode shows giant Kohn anomalies at $2 k_{F}$ for the inner and outer bands.

It is important to realize that the divergence of $\Omega_{q \mu}$ shown in Fig. 2 does not imply the divergence of $\Omega_{q \mu}^{0}$ in 


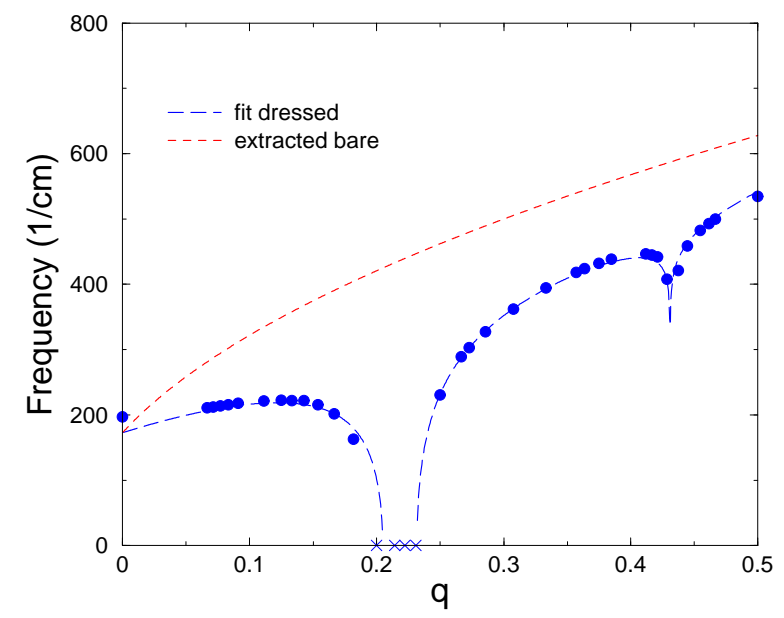

FIG. 2: The phonon dispersion for the out-of-plane optical mode in the $(5,0) \mathrm{CNT}$ showing logarithmic divergences at $2 k_{F}$. We show the fit to the out-of-plane optical mode for which the bare frequencies are extracted as discussed in the text. The X's denote values of $q$ at which the calculated FPA frequencies were imaginary.

the Frölich Hamiltonian Eq. (11). In the FPA, the phonon frequencies are calculated after the electron-phonon interaction in Eq. (11) have been included, which gives anomalous softening at $2 k_{F}$ due to the well-known Peierls instability of electron-phonon systems in 1d. We have developed a technique to extract the bare phonon frequency $\Omega_{q \mu}^{0}$ from the numerically computed $\Omega_{q \mu}$ using a connection between the frozen-phonon approximation and the RPA treatment of the giant Kohn anomaly which is articulated in Ref. 17. Briefly, the dressed phonon frequencies $\Omega_{q \mu}$ and the bare phonon frequencies $\Omega_{q \mu}^{0}$ satisfy the equation

$$
\left(\Omega_{q \mu}\right)^{2}=\left(\Omega_{q \mu}^{0}\right)^{2}+2 \Omega_{q \mu}^{0} \Pi_{\mu}(q)
$$

where $\Pi_{\mu}(q)$ is the phonon self-energy. Using the calculated FPA values for $\Omega_{q \mu}$, and the calculated electronphonon coupling values to determine $\Pi_{\mu}(q)$ (in the random-phase approximation), we can extract the bare phonon frequencies by assuming that they have the form $\Omega_{q \mu}^{0}=A+B q+C q^{2}$ and performing least-squares fitting where $A, B$, and $C$ are adjustable parameters. Using this method, we have calculated the bare phonon frequencies of the representative nanotubes, thereby constructing the effective Frölich Hamiltonians for these systems.

Now we consider possible instabilities of the electronphonon system. Using the RPA analysis on the Frölich Hamiltonian, we find the CDW transition temperature is given by

$$
T_{\mathrm{CDW}}=4 \varepsilon_{\mathrm{F}} e^{-1 / \lambda_{\mathrm{CDW}}} .
$$

Here, $\lambda_{\mathrm{CDW}}$ is the dimensionless CDW coupling constant given by $\lambda_{\mathrm{CDW}}=\left|g_{q \mu}\right|^{2} \nu_{\tau}(0) / \Omega_{q \mu}^{0}$ where $\nu_{\tau}(0)$ is the density of states per spin at the Fermi energy of the band

\begin{tabular}{l|c|c|c|} 
& $(5,0)$ & $(6,0)$ & $(5,5)$ \\
\hline$\lambda_{\mathrm{CDW}}$ & 0.26 & 0.12 & 0.024 \\
$\lambda_{\mathrm{SC}}$ & 0.57 & 0.12 & 0.031 \\
$\mu_{\mathrm{CDW}}$ & 0.24 & 0.0019 & 0.013 \\
$\mu_{\mathrm{SC}}^{*}$ & 0.19 & 0.16 & 0.093 \\
$T_{\mathrm{CDW}}^{0}(\mathrm{~K})$ & 160 & 5.0 & $7 \times 10^{-14}$ \\
$T_{\mathrm{SC}}^{0}(\mathrm{~K})$ & 64 & 0.071 & $1 \times 10^{-12}$ \\
$T_{\mathrm{CDW}}(\mathrm{K})$ & $1 \times 10^{-18}$ & 5.0 & $2 \times 10^{-43}$ \\
$T_{\mathrm{SC}}(\mathrm{K})$ & 1.1 & - & -
\end{tabular}

TABLE I: The CDW and SC coupling constants and transition temperatures for the CNTs studied. $\lambda_{\mathrm{SC}}$ does not include Coulomb screening or the temperature dependent softening of phonons discussed in the text. $T_{\mathrm{CDW}, \mathrm{SC}}^{0}$ was computed without the residual Coulomb interaction while the residual Coulomb interaction is included in the calculation of $T_{\mathrm{CDW}, \mathrm{SC}}$. $T_{\mathrm{SC}}$ includes the temperature dependent renormalization of $\lambda_{\mathrm{SC}}$ (see Eq. (12)).

that is undergoing the transition. In table [1] we summarize the results for the CDW coupling constants and transition temperatures for the nanotubes we study. The leading CDW instability for the $(5,0)$ and $(6,0)$ CNTs is from the out-of-plane optical phonon mode which is shown in Fig. 1 while the leading CDW instability for the $(5,5)$ CNT is to an in-plane optical mode.

To study superconductivity, we use the McMillan formula

$$
T_{\mathrm{SC}}=\frac{\langle\Omega\rangle}{1.20} \exp \left[-\frac{1.04\left(1+\lambda_{\mathrm{SC}}\right)}{\lambda_{\mathrm{SC}}-\mu_{\mathrm{SC}}^{*}\left(1+0.62 \lambda_{\mathrm{SC}}\right)}\right] .
$$

where the dimensionless superconducting coupling constant is given by

$$
\lambda=\frac{1}{\nu(0)} \sum_{k \tau k^{\prime} \tau^{\prime} \mu} \delta\left(\varepsilon_{k \tau}\right) \delta\left(\varepsilon_{k^{\prime} \tau^{\prime}}\right)\left|g_{k \tau k^{\prime} \tau^{\prime} \mu}\right|^{2} \frac{2}{\Omega_{q \mu}} .
$$

In the above equations, $\nu(0)$ is the density of states at the Fermi energy per spin, $\langle\Omega\rangle=1400 \mathrm{~K}[19]$ is the logarithmically averaged phonon frequency, and $\mu_{\mathrm{SC}}^{*}$ is the Coulomb pseudopotential which we will set to zero for the time being. The results for the superconducting coupling constants and transition temperatures is also summarized in table \for the nanotubes we study.

From this analysis, we find that both the CDW and $\mathrm{SC}$ instabilities for the $(5,5)$ nanotube occur below experimentally realizable temperatures. This leads one to expect that conventional CNTs of larger radius also be stable down to very low temperatures. For the $(5,0)$ and $(6,0)$ CNTs, the CDW instability was found to be dominant which occurs from coupling to the out-of-plane optical phonon mode.

Now we consider the consequences of introducing the residual Coulomb interaction. We point out that since the charge density is not evaluated self-consistently in the tight binding method we use, the Hartree term which 
opposes the formation of the charge-density wave is omitted in our frozen-phonon calculation of frequencies. This term essentially gives the Coulomb energy cost of forming a non-uniform charge density. Including the Coulomb interactions properly should lessen the divergences found at $2 k_{F}$ in the phonon spectra. Introducing the residual Coulomb interaction changes our effective Hamiltonian to

$$
\mathcal{H}=\mathcal{H}_{\mathrm{e}-\mathrm{ph}}+\mathcal{H}_{\mathrm{e}-\mathrm{e}}
$$

where

$$
\mathcal{H}_{\mathrm{e}-\mathrm{e}}=\frac{1}{2} \sum_{k k^{\prime} q \tau \tau^{\prime} \sigma \sigma^{\prime}} V_{q \tau \tau^{\prime}} c_{k+q \tau \sigma}^{\dagger} c_{k^{\prime}-q \tau^{\prime} \sigma^{\prime}}^{\dagger} c_{k^{\prime} \tau^{\prime} \sigma^{\prime}} c_{k \tau \sigma}
$$

and $\mathcal{H}_{\mathrm{e}-\mathrm{ph}}$ is given by Eq. (1). For the Coulomb interaction between conduction electrons, we take the form used by Egger et al. in Ref. 15 which, in position space, is given by

$$
V\left(\mathbf{r}-\mathbf{r}^{\prime}\right)=\frac{e^{2} / \kappa}{\sqrt{\left(x-x^{\prime}\right)^{2}+\left(2 R \sin \left(\frac{y-y^{\prime}}{2 R}\right)\right)^{2}+a_{z}^{2}}} .
$$

Here, the $y$-direction is chosen to be along the perimeter of the CNT and $x$ measures the distance along the CNT axis. A measure of the spatial extent of the $p_{z}$ electrons perpendicular to the CNT is given by $a_{z} \approx 1.6 \AA$ and $R$ is the CNT radius. For the dielectric constant due to the bound electrons, we will take the value $\kappa \approx 2$ predicted by the model of Ref. [20].

Including the Coulomb interaction in our RPA analysis of the CDW instability, we find that the transition temperature is modified to

$$
T_{\mathrm{CDW}}=4 \varepsilon_{\mathrm{F}} e^{-1 /\left(\lambda_{\mathrm{CDW}}-\mu_{\mathrm{CDW}}\right)}
$$

where $\mu_{\mathrm{CDW}}=\nu_{\tau}(0) V_{q=2 k_{F}}$ and $\nu_{\tau}(0)$ is the density of states per spin at the Fermi energy for the band that is undergoing the instability. We thus find that the effective coupling is directly reduced by including the Coulomb interaction.

With our model for the Coulomb interaction, we find for the $(5,0)$ CNT that $\mu_{\mathrm{CDW}}=0.24$ which dramatically reduces $T_{\mathrm{CDW}}$ to around $10^{-18} \mathrm{~K}$. Thus the CDW instability for the $(5,0) \mathrm{CNT}$ is essentially removed by taking into account the residual Coulomb interaction between conduction electrons. The case is somewhat different for the $(6,0) \mathrm{CNT}$, however. For such metallic zigzag nanotubes, the wave functions at $-k$ and $k$ close to the Fermi energy correspond to symmetric and antisymmetric combinations of atomic orbitals in the graphene sheet [17]. Orthogonality of these wave functions within the unit cell of the CNT leads to the significantly smaller $\mu_{\mathrm{CDW}}=0.0019$ which essentially does not affect the CDW transition temperature.
The residual Coulomb interaction comes up in more subtle ways when considering the superconducting instability. By properly dressing the electron-phonon vertices as well as the phonon propagator in Migdal's expression for the electronic self-energy we find that the renormalized contribution to the superconducting coupling constant is given by

$$
\lambda_{q \mu}=\left(\frac{1}{\left(1-V_{q} \chi_{0}(q)\right)^{2}}\right)\left(\frac{1}{1+\frac{2\left|g_{q \mu}\right|^{2}}{\Omega_{q \mu}^{0}} \frac{\chi_{0}(q)}{1-V_{q} \chi_{0}(q)}}\right) \lambda_{q \mu}^{0}
$$

where $\lambda_{q \mu}^{0}$ is the unrenormalized contribution for a specific process of wave vector $q$ coupling points on the Fermi surface by phonon mode $\mu$ and $\chi_{0}(q)=\sum_{k}\left(f_{k+q}-\right.$ $\left.f_{k}\right) /\left(\varepsilon_{k+q}-\varepsilon_{k}\right)$ [17]. The first factor describes renormalization of the electron-phonon vertex by Coulomb interaction and tends to decrease $T_{\mathrm{SC}}$ while the second factor corresponds to phonon softening due to the giant Kohn anomaly in $1 \mathrm{~d}$ which tends to increase $T_{\mathrm{SC}}$. The temperature dependence described by Eq. (12) is similar to the two parameter RG analysis presented in Ref. [21]. In addition to the renormalization of $\lambda_{\mathrm{SC}}$ we also have the direct repulsion between conduction electrons which is taken into account through the Coulomb pseudopotential as shown in the McMillan formula 6] Analysis based on Eq. (10) we find that the inclusion of $\mu_{\mathrm{SC}}^{*}$ eliminates superconductivity in the $(5,5)$ and $(6,0)$ CNTs [17]. For the $(5,0) \mathrm{CNT}$, the main contribution to the renormalization of $\lambda_{\mathrm{SC}}$ comes the $2 k_{F}$ coupling to the out-of-plane optical mode discussed earlier. Taking into account the temperature dependence in $\chi_{0}(q)$, using Eqns. (6) and (12) we find a self-consistent solution of $T_{\mathrm{SC}}=1.1 \mathrm{~K}$. Thus we see that inclusion of the Coulomb interactions makes superconductivity dominant over the CDW in ultrasmall $(5,0)$ CNTs. We point out that our estimates give a meanfield value of $T_{\mathrm{SC}}$. Below this temperature we expect a gradual decrease of resistivity, which may be described by the Langer-Ambegaokar-McCumber-Halperin formalism [22, 23]. Discrepancy between this value of $T_{\mathrm{SC}}$ and the one observed experimentally of $15 \mathrm{~K}$ [7] should not be a reason for concern because of the exponential dependence of the superconducting transition temperature on the Coulomb interaction strength and the known difficulty in calculating the latter accurately. For instance, if we replace our estimated value of $\mu_{\mathrm{SC}}^{*}=0.19$ by the commonly used $\mu_{\mathrm{SC}}^{*}=0.10$, we find a self-consistent solution for the superconducting transition temperature for the $(5,0) \mathrm{CNT}$ of $T_{\mathrm{SC}}=13 \mathrm{~K}$.

We thank S. Kivelson, I. Mazin, M. Mehl, M. Tinkham, and especially B. Halperin for very useful discussions. This work was supported by Harvard NSEC and by the Sloan foundation. RB was supported by an NSF graduate research fellowship. 
[1] S. Ijima, Nature 54, 56 (1991).

[2] R. Saito, G. Dresselhaus, and M. S. Dresselhaus, Physical Properties of Carbon Nanotubes (Imperial College Press, London, 1998).

[3] R. Egger, A. Bachtold, M. Fuhrer, and M. Bockrath, in R. Haug and H. Schoeller, eds., Interacting Electrons in Nanostructures (Springer Verlag, 2001).

[4] D. H. Cobden, M. Bockrath, P. L. McEuen, A. G. Rinzler, and R. E. Smalley, Phys. Rev. Lett. 81, 681 (1998).

[5] J. Nygard, D. Cobden, and P. Lindelof, Nature 408, 342 (2000).

[6] M. Kociak, A. Y. Kasumov, S. Gueron, B. Reulet, I. I. Khodos, Y. B. Gorbatov, V. T. Volkov, L. Vaccarini, and H. Bouchiat, Phys. Rev. Lett. 86, 2416 (2001).

[7] Z. Tang, L. Zhang, N. Wang, X. Zhang, G. Wen, G. Li, J. Wang, C. Chan, and P. Sheng, Science 292, 2462 (2001).

[8] T. Hertel and G. Moos, Phys. Rev. Lett. 84, 5002 (2000).

[9] J.-Y. Park, S. Rosenblatt, Y. Yaish, V. Sazonova, H. Ustunel, S. Braig, T. A. Arias, P. W. Brouwer, and P. L. McEuen (2004), condmat/0309641.

[10] J. W. Mintmire, B. I. Dunlap, and C. T. White, Phys. Rev. Lett. 68, 631 (1992).

[11] O. Dubay, G. Kreese, and H. Kuzmany, Phys. Rev. Lett.
88, 235506 (2002).

[12] A. DeMartino and R. Egger, Phys. Rev. B 67, 235418 (2003).

[13] X. Blase, L. X. Benedict, E. L. Shirley, and S. G. Louie, Phys. Rev. Lett. 72, 1878 (1994).

[14] Z. M. Li, Z. K. Tang, H. J. Liu, N. Wang, C. T. Chan, R. Saito, S. Okada, G. D. Li, J. S. Chen, N. Nagasawa, et al., Phys. Rev. Lett. 87, 127401 (2001).

[15] R. Egger and A. O. Gogolin, Eur. Phys. J. B 3, 281 (1998).

[16] M. J. Mehl and D. A. Papaconstantopoulos, Phys. Rev. B 54, 4519 (1996).

[17] R. Barnett, E. Demler, and E. Kaxiras, condmat/0305006.

[18] M. T. Yin and M. L. Cohen, Phys. Rev. B 26, 5668 (1982).

[19] L. X. Benedict, V. H. Crespi, S. G. Louie, and M. L. Cohen, Phys. Rev. B 52, 14935 (1995).

[20] L. X. Benedict, V. H. Crespi, S. G. Louie, and M. L. Cohen, Phys. Rev. B 52, 8541 (1995).

[21] G. S. Grest, E. Abrahams, S.-T. Chui, P. A. Lee, and A. Zawadowski, Phys. Rev. B 14, 1225 (1976).

[22] J. S. Langer and V. Ambegaokar, Phys. Rev. 164, 498 (1967).

[23] D. E. McCumber and B. I. Halperin, Phys. Rev. B 1, 1054 (1970). 